\title{
REPRESENTAÇÕES SOCIAIS SOBRE SISTEMATIZAÇÃO DA ASSISTÊNCIA À CRIANÇA HOSPITALIZADA
}

\author{
Carolina Sampaio de Oliveira ${ }^{1}$, Moema da Silva Borges ${ }^{2}$
}

RESUMO: A pesquisa teve como objetivo conhecer o núcleo central das representações sociais dos enfermeiros que prestam assistência à criança sobre a Sistematização da Assistência de Enfermagem. Trata-se de estudo exploratório-descritivo de natureza qualitativa, com enfoque na fundamentação teórico-metodológica da Teoria da Abordagem Estrutural da Teoria das Representações Sociais, com coleta de dados em maio de 2015. Participaram do estudo 45 enfermeiros que atuavam no cuidado à criança em dois hospitais escola do estado do Mato Grosso. Aplicou-se uma entrevista utilizando-se a técnica de Associação Livre de Palavras que foi analisada com auxílio do software Evoc 2000. Os resultados revelam que os termos "cuidado", "enfermagem" e "organização" foram os mais frequentemente evocados figurando como o provável núcleo central da representação social.

DESCRITORES: Assistência de enfermagem; Cuidados de enfermagem; Saúde da criança; Cuidado da criança; Humanização da assistência.

\section{SOCIAL REPRESENTATIONS ON SYSTEMATIZATION OF CARE TO HOSPITALIZED CHILDREN}

ABSTRACT: The present study aimed to obtain insight on the core of social representations of nurses who provide care to children on the Systematization of Nursing Care. This is an exploratory and descriptive study of a qualitative nature, focused on the theoreticalmethodological foundation of Structural Approach, applied to the Theory of Social Representations, and data was collected in May 2015. Participants were 45 nurses caring for children in two university hospitals in the state of Mato Grosso. An interview based on the word association test was administered and then analyzed using software Evoc 2000. The results showed that the words "care", "nursing" and "organization" were the most frequently mentioned and therefore, are likely to be the central core of social representation.

DESCRIPTORS: Nursing assistance; Nursing care; Child health. Child care; Humanization of care.

\section{REPRESENTACIONES SOCIALES ACERCA DE LA SISTEMATIZACIÓN DE LA ASISTENCIA AL NIÑO HOSPITALIZADO}

RESUMEN: Investigación cuyo objetivo fue conocer el núcleo central de las representaciones sociales de enfermeros que atienden a niños en lo que se refiere a la Sistematización de la Asistencia de Enfermería. Es un estudio exploratorio y descriptivo de naturaleza cualitativa, con énfasis en la fundamentación teórica metodológica de la Teoría del Abordaje Estructural de la Teoría de las Representaciones Sociales. Los datos fueron obtenidos en mayo de 2015. Participaron del estudio 45 enfermeros que tenían como oficio el cuidado al niño en dos hospitales de enseñanza del estado de Mato Grosso. Fue utilizada entrevista por medio de técnica de Asociación Libre de Palabras, la cual fue analizada con ayuda del software Evoc 2000. Los resultados muestran que los términos "cuidado", "enfermería" y "organización" fueron los evocados con más frecuencia, presentándose como probable núcleo central de la representación social.

DESCRIPTORES: Asistencia de enfermería; Cuidados de enfermería; Salud del niño; Cuidado del niño; Humanización de la asistencia.

${ }^{1}$ Enfermeira. Doutoranda em Enfermagem. Docente de Enfermagem da Universidade do Estado de Mato Grosso. Cáceres, MT, Brasil.

${ }^{2}$ Enfermeira. Doutora em Ciências da Saúde. Docente do Programa de Pós Graduação em Enfermagem da Universidade de Brasília. Brasília, DF, Brasil. 


\section{- INTRODUÇÃO}

O cuidar na enfermagem compreende as ações desenvolvidas com competência e tem o objetivo de favorecer as potencialidades das pessoas, no sentido de manter ou melhorar sua condição humana no processo de viver e morrer ${ }^{(1)}$. Para tal, utiliza-se um modelo de organização do trabalho pautado no conhecimento, que sistematiza as ações, permitindo reflexão, criticidade e segurança, denominado Sistematização da Assistência de Enfermagem (SAE), operacionalizado especialmente pelo Processo de Enfermagem $(\mathrm{PE})^{(2)}$.

A SAE se propõe a organizar o trabalho da equipe de enfermagem em todos os níveis de atenção à saúde proporcionando ao paciente, família ou comunidade a prática integral, individual e humanizada que propicia também maior eficiência, autonomia e cientificidade à profissão, garantindo maior valorização e reconhecimento. Representa espaço de novas conquistas e pode significar o início para mudança cultural no papel do Enfermeiro ${ }^{(3)}$.

Nesse contexto, a aplicação do cuidado sistematizado na assistência à criança constitui ferramenta de planejamento indispensável, uma vez que as singularidades da infância demandam maior complexidade, sensibilidade e organização do enfermeiro e abordagem que agregue ao cuidado de saúde a necessidade do brincar, e a resolutividade dos processos por meio de redes de apoio em saúde $^{(4)}$.

Assim, o cuidado infantil deve considerar o processo de crescimento e desenvolvimento, as diferenças anatomofisiológicas do organismo, o desenvolvimento cognitivo de cada faixa etária, o processo de comunicação entre quem cuida e quem é cuidado, além das relações e inter-relações das famílias, equipes e crianças $^{(5)}$.

Nesta perspectiva, a vinculação da SAE à prática clínica da pediatria oferece ao enfermeiro a possibilidade de diagnosticar situações de sua competência, proporcionando evidências para embasar suas ações, apontar e justificar a seleção de determinados problemas, e direcionar as atividades de cada um dos integrantes de sua equipe ${ }^{(2)}$.

Apreender como o enfermeiro percebe e estabelece afinidades com este método científico, como os conceitos são construídos, reconstruídos e fortalecidos diariamente, é de fundamental importância para compreender quais são os investimentos e estímulos necessários para influenciar positivamente o desenvolvimento e aplicação da SAE no ambiente de cuidado à criança.

O que se espera é qualificar a assistência e valorizar as Representações Sociais (RS) sobre a prática profissional, sabendo-se que as RS se originam no cotidiano, por meio de comunicações interindividuais que contribuem para a formação de condutas. Assim, representar algo consiste não somente em descrevê-lo ou reproduzi-lo, é reconstruí-lo, modificá-lo a partir de trocas, interações e experiências humanas ${ }^{(6)}$.

Nesse contexto, RS é considerada um conjunto organizado e estruturado de informações, crenças, opiniões e atitudes, dispostas em dois sistemas, o núcleo central e o sistema periférico, que funcionam como duplo sistema em que cada parte tem um papel específico e complementar ${ }^{(7)}$.

O sistema ou núcleo central está relacionado à memória coletiva, é dado pela análise da história de vida de determinado grupo e suas experiências, o que corresponde a suas bases histórica, social e psicológica. Devido a esse aspecto, consegue dar significado, consistência e permanência à representação, aferindo o caráter de estabilidade à representação e marca a resistência às mudanças de pensamento de determinado grupo ${ }^{(7)}$.

O sistema periférico é responsável pela atualização e contextualização da representação, permite que a representação se adapte ao discurso do momento ${ }^{(7)}$. Sendo assim, os elementos do sistema periférico são mais vivos, mais concretos e de grande mobilidade. Embora o sistema periférico não defina a RS, ele contribui efetivamente para sua organização(8).

Diante do exposto, o objetivo deste estudo foi apreender o núcleo central das RS sobre a SAE para enfermeiros que cuidam de crianças hospitalizadas. Pretende-se estimular a reflexão sobre a importância do emprego da SAE como método sistemático e operacional no planejamento das 
ações de enfermagem, bem como fortalecer o conhecimento próprio da categoria, favorecendo a ressignificação da prática assistencial à criança.

\section{MÉTODO}

Tratou-se de estudo exploratório-descritivo de natureza qualitativa, com enfoque na fundamentação teórico-metodológica da Teoria da Abordagem Estrutural das Representações Sociais, de Jean-Claude Abric $^{(7)}$.

Participaram do estudo enfermeiros que atuavam na assistência à criança de dois hospitais-escola de um município do Estado de Mato Grosso que prestam atendimento voltado para o Sistema Único de Saúde (SUS), são referências para o atendimento infantil e campos de instrumentalização prática para três instituições de ensino superior. Vale ressaltar que esses dois hospitais implantaram a SAE desde o ano 2000.

Nos hospitais foram selecionados, como espaços específicos de pesquisa, apenas os setores que prestavam algum nível de assistência às crianças, a saber: pediatria, unidade de terapia intensiva (UTI) neonatal, UTI pediátrica, pronto atendimento infantil, box, sala de parto e centro cirúrgico.

Os critérios de inclusão dos enfermeiros foram: estar lotados nos setores selecionados, e atuar neste mesmo setor há mais de seis meses. A escolha deste critério buscou privilegiar maior contato com a vivência e a experiência da realidade dos profissionais nos referidos espaços. Foram excluídos os enfermeiros que trabalhavam no sistema de plantão esporádico. Constatou-se que no total atuavam nesses espaços 56 enfermeiros, com média de quatro enfermeiros por cenário.

A coleta dos dados foi realizada em maio de 2015, por meio de entrevista em sala reservada, disponibilizada pelos hospitais para essa finalidade, após prévio agendamento com cada um dos enfermeiros.

Foram utilizados dois instrumentos. O primeiro era composto por perguntas que objetivaram desenhar o perfil dos participantes; e o segundo deu suporte para o emprego da técnica de livre associação de palavras, que se consistiu em apresentar a expressão indutora "Sistematização da Assistência de Enfermagem", solicitando aos participantes a produção e a posterior classificação, por ordem de prioridade, de três palavras, expressões e/ou adjetivos que lhe viessem à mente.

Os dados obtidos foram transcritos e analisados com o auxílio do software Evoc 2000. As palavras evocadas com maior frequência devem constituir o provável núcleo central da representação, e as palavras com menores frequências o sistema periférico da representação ${ }^{(9)}$.

Sendo assim, o uso do software Evoc 2000 permitiu demarcar o provável núcleo central e o sistema periférico em função de duplo critério: frequência e ordem de evocação das palavras. A partir do cruzamento dos critérios de frequência e evocação, foi definida a relevância dos elementos associados (palavras, frases e expressões) ao termo indutor. Esses resultados são apresentados em um quadro com quatro quadrantes, organizados em dois eixos. O eixo vertical contempla a frequência, enquanto o horizontal, a ordem de evocação ${ }^{(9)}$.

No quadrante superior esquerdo do quadro, aparecem os elementos mais relevantes que surgem nos primeiros lugares da ordem de evocação, com uma frequência significativamente mais elevada (núcleo central). Nos quadrantes superior e inferior direito desse quadro são contempladas as palavras menos relevantes quanto ao seu papel na estrutura da representação, embora significativos em sua organização (sistema periférico) ${ }^{(9)}$.

Os dados foram coletados após aprovação do projeto de pesquisa, em 14 de maio de 2015, pelo Comitê de Ética da Universidade Estadual de Mato Grosso, sob parecer 1.065.610. Os participantes do estudo, após concordarem em participar da pesquisa, assinaram o Termo de Consentimento Livre e Esclarecido. 


\section{RESULTADOS}

Após aplicação dos critérios de inclusão e exclusão, a amostra do estudo foi composta por 45 enfermeiros, cujo perfil foi constituído da seguinte forma: 29 entrevistados tinham entre 22 e 29 anos; 15 tinham entre 30 e 37 anos; e dois tinham entre 38 e 45 anos. Quanto ao sexo, 42 dos entrevistados eram do sexo feminino e três do sexo masculino. Quanto à instituição formadora, 28 realizaram o curso em instituição pública estadual do município da pesquisa, três em instituição pública federal do estado onde a pesquisa foi realizada, 12 em instituições particulares do estado de Mato Grosso e dois cursaram em universidades federais fora do Estado de Mato Grosso. Dentre os enfermeiros, 17 eram graduados e 28 eram especialistas. Quanto ao tempo utilizado para finalização do curso de Enfermagem, três participantes o fizeram em 4 anos e 39 em 5 anos.

A análise do corpus formado pelas evocações dos 45 participantes, nos dois hospitais-escola, revelou que, em resposta ao termo indutor "Sistematização da Assistência de Enfermagem", foram evocadas 135 palavras e, destas, foram selecionados pelo software 13 termos apresentando média da ordem de evocação de 2,0. Foram desprezadas as evocações cuja frequência foi inferior a 3; a frequência média foi de 8 evocações. O quadro de quatro casas apresentado na Figura 1 foi resultante da análise combinada desses indicadores (frequência e ordem de evocação).

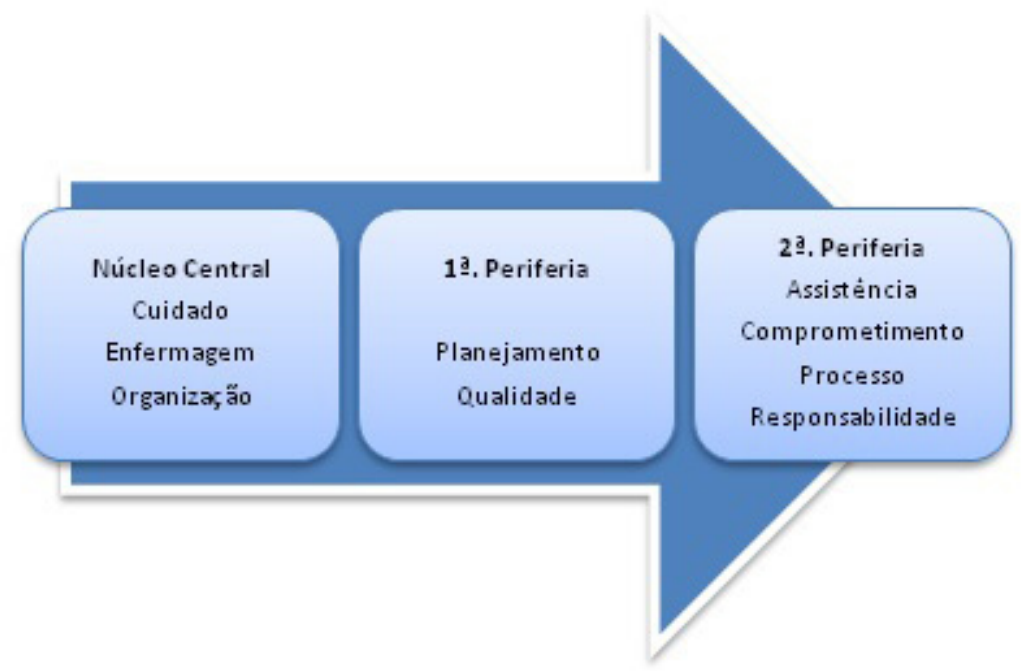

Figura 1 - Principais termos evocados pelos entrevistados após o estímulo indutor "Sistematização da Assistência de Enfermagem". Cáceres, MT, Brasil, 2015

O sistema periférico desta representação é formado por duas periferias, constituídas de palavras que organizam a representação do núcleo central. As palavras da primeira e segunda periferia seguem a moda, ou o discurso corrente no momento da coleta de dados. Constatou-se que foram formados por palavras que tinham sua significação atrelada às características desenhadas para a construção do perfil ideal do contemporâneo profissional de enfermagem, salientando os benefícios da aplicação do cuidado sistematizado.

A análise dos resultados dos questionários de livre associação de palavras permitiu apreender os valores, as crenças e a prática dos participantes diante da expressão indutora, percebendo os termos formadores do provável núcleo central (quadrante superior esquerdo) desta representação e do sistema periférico (quadrante superior e inferior direito) (Quadro 1). 
Quadro 1 - Distribuição das representações sociais para o estímulo indutor Sistematização da Assistência de Enfermagem. Cáceres, MT, Brasil, 2015

\begin{tabular}{|l|l|c|c|l|c|c|}
\hline${ }^{*}$ OMI & $\mathbf{< 2 , 0}$ & & & $\mathbf{2}, \mathbf{0}$ & & \\
\hline Frequência Média & Núcleo central & $\mathbf{F * *}$ & Rang+ & Elementos periféricos 1 & F & Rang \\
\hline & Cuidado & 17 & 1,765 & Planejamento & 8 & 2,5 \\
\hline & Enfermagem & 10 & 1,6 & Qualidade & 17 & 2,059 \\
\hline$\geq 8$ & Organização & 13 & 1,538 & & & \\
\hline & Elementos de contraste & $\mathbf{F}$ & Rang & Elementos periféricos 2 & $\mathbf{F}$ & Rang \\
\hline $3 \leq$ & Ciência & 3 & 1,2 & Assistência & 4 & 2 \\
\hline & Humanização & 3 & 1,667 & Comprometimento & 4 & 2 \\
\hline$<7$ & Obrigação & & & Responsabilidade & 3 & 2,5 \\
\hline & & & Processo & 4 & 2 \\
\hline
\end{tabular}

* Ordem média das evocações; **frequência das evocações; † distribuição das evocações.

\section{DISCUSSÃO}

Frente à expressão indutora "Sistematização da Assistência de Enfermagem", os sujeitos evocaram mais prontamente as palavras: cuidado, enfermagem e organização. Estas palavras tiveram as maiores frequências, indicando os elementos que constituem o núcleo central desta representação e refletem a dimensão das ações de enfermagem para estes profissionais, ou seja, traduzem o entendimento do cuidado organizado como objeto de trabalho da enfermagem.

O termo "cuidado" obteve maior frequência de evocação, apontando ser o provável núcleo central das representações sociais acerca da SAE.

O cuidado de enfermagem é universal, multidimensional, singular e deve considerar os sistemas social, econômico e cultural dos envolvidos. Assim, a atuação da enfermagem está compreendida em uma zona de intercessão entre o cuidado e o tratamento. Embora esses possam ser confundidos com a aplicação de uma técnica, são, na verdade, frutos de um conjunto de ações que se baseiam nas leis que presidem a saúde, com as quais se confundem, porque são da mesma natureza e essenciais, tanto para a manutenção quanto para a reparação da vida ${ }^{(10)}$.

Nessa perspectiva, o cuidado de enfermagem pode ser explicado como um conjunto de ações desenvolvidas pelo profissional de enfermagem em substituição dos cuidados que a pessoa não pode temporariamente garantir a si própria, ou que não pode ser garantido por seu entorno social ${ }^{(10)}$. A este termo reforça a concepção do ser enfermeiro, expressando sua ciência e seu objeto de trabalho.

O cuidado ao atendimento de saúde da criança foi, durante muitos anos, pautado na cura da doença. Atualmente, está em construção um modelo assistencial em que a criança deixa de ser compreendida como ser unicamente biológico, que privilegia cuidado assistencial centrado em suas especificidades e de sua família, mediante a construção de redes de apoio, buscando sua integralidade ${ }^{(11)}$.

Nesta nova perspectiva, o cuidado de enfermagem deve promover e restaurar o bem-estar físico, psíquico e social, considerando aspectos intrínsecos ligados à sua condição de criança e reconhecendo o protagonismo familiar, bem como as legislações que garantem suas especificidades ${ }^{(12-13)}$.

Desta forma, ao considerar o cuidado como o possível núcleo central da RS da SAE, os participantes reconhecem que o cuidado constitui a verdadeira competência da enfermagem. Traduz uma ação que vai além de procedimentos técnicos, englobando o envolvimento e o compromisso com o outro e, portanto, uma ação humanizada, que melhora as condições e favorece a promoção e a prevenção da saúde, objetivando limitar a doença e promover a vida ${ }^{(14)}$.

Quando profissionais da enfermagem respondem às necessidades de cuidado e agem responsavelmente, ajudando a desenvolver, restaurar ou ampliar o cuidado na melhor maneira 
possível, temos o cuidado autêntico. Ao agir assim, os pacientes não são apenas ajudados a lidar e a enfrentar a doença e a incapacidade, mas são encorajados e empoderados a continuar em sua busca pela plenitude humana ${ }^{(1)}$.

O segundo estímulo respondido, também como provável termo associado ao núcleo central da RS da SAE, com frequência de apresentação igual a dez e que fortalece as discussões relacionadas ao cuidado, foi a palavra Enfermagem. Deve-se ressaltar que a Enfermagem moderna tem predominância do modelo humanístico e suas práticas profissionais foram historicamente marcadas pela forte influência do espírito religioso, da organização militar, dos princípios da divisão social do trabalho e, mais recentemente, pela busca de conhecimento próprio ${ }^{(15)}$.

É, por essência, desde o seu nascimento, expressa através do cuidado, e este cuidado durante todo o processo de formação do enfermeiro é abordado dentro de uma dimensão essencial e complexa, tanto na experiência de quem cuida quanto de quem recebe o cuidado, ou até mesmo de quem ensina a cuidar ${ }^{(3)}$.

A profissão de enfermagem é regulamentada no território brasileiro pela lei 7.498/86, composta por três classes profissionais com atribuições específicas e proporcionais aos níveis de complexidade assistencial e ao tempo de formação de cada categoria ${ }^{(16)}$. As inúmeras atribuições desenvolvidas por essa profissão abrangem afazeres técnicos e procedimentais, abordando características pessoais, como liderança, humanização, competência gerencial e inovação, bem como o desenvolvimento de boas relações pessoais ${ }^{(17)}$.

Convém ressaltar que o cuidado dispensado pelo enfermeiro à criança envolve também seus familiares e sua equipe, e seu singular significativo, pois somente por meio desta dinâmica é possível garantir melhor relacionamento, interdependência, coesão e competência nas ações ${ }^{(13-14)}$.

Desse modo, a enfermagem contemporânea une a arte-ciência-ideal do cuidar, possibilitando ações que beneficiam o resgate do ser humano integral, fortalecendo os vínculos entre o sentir-saberfazer $^{(15)}$. Assim, a enfermagem assume a proposta de abordagem global do indivíduo, estabelecendo-se como prática de cuidado integralizadora.

A terceira palavra encontrada, relacionada à formação do núcleo central desta representação, foi o termo "organização". Considerando o cuidado a marca e o núcleo de atenção da enfermagem, é preciso compreender que, para cuidar, são necessários instrumentos e processos metódicos, ou seja, de organização, para que o desfecho da ação base possa ser considerado integral e resolutivo.

As ações provenientes da rotina do enfermeiro devem estar voltadas para a qualidade do cuidado, de forma a abraçar as dimensões assistencial e gerencial desta prática. Uma ação cuidadora responsável perpassa pela avaliação e pelo planejamento de todas as condições que envolvem determinada intervenção, ou seja, a organização da assistência, através da adoção de determinado método e o embasamento em conhecimentos científicos ${ }^{(18)}$.

Apreendendo os três termos com maior frequência de evocação, "cuidado", "enfermagem" e "organização", e que por esta razão constituem o núcleo central desta representação, podemos observar que estes são congruentes e convergem para o termo indutor da pesquisa: "Sistematização da Assistência de Enfermagem".

Tratando-se de representação do núcleo central, ou seja, daquela que se mantém resistente a mudanças, pode-se inferir que a representação analisada reflete a premissa básica das práticas de enfermagem proposta por diversos referenciais da área. Ou seja, a enfermagem é uma profissão que necessita de modelo de organização, para administrar os diversos recursos necessários para o desenvolvimento do cuidado.

Portanto, a SAE no cuidado à criança constitui um arquétipo teórico para a prática assistencial integral, fazendo com que a atuação dos profissionais de enfermagem seja cientificamente coerente, planejada quanto à identificação de necessidades da criança e sua família, obtendo estabelecimento, alcance de metas e avaliação.

Formando o sistema periférico desta RS temos a primeira e a segunda periferia. Na primeira periferia, (quadrante superior direito), a presença dos termos "planejamento" e "qualidade" remete à dimensão 
avaliativa do cuidado e expressa posicionamento positivo diante da assistência de enfermagem. Os termos evocados se apresentam bem próximos ao núcleo central, já que contam com frequência de análise e ordem de evocação próximas da média do grupo.

O sistema periférico é formado por elementos mais dinâmicos e passíveis de atualizações; relacionado às características individuais e ao contexto; adaptando-se às situações específicas e contribuindo para sua organização ${ }^{(6)}$. Pode-se inferir, então, que os conceitos de planejamento e qualidade constituem complementos indispensáveis do sistema central, do qual ele depende, ou seja, o cuidado sistematizado depende de planejamento e leva à qualidade.

A palavra "planejamento" está presente com frequência alta, reforçando a atitude positiva da ação diante do desenvolvimento do cuidado de enfermagem.

As manifestações das palavras evocadas estão em consonância com a raiz essencial do termo "Sistematização da Assistência de Enfermagem" e fortalecem a importância desta para o desenvolvimento do cuidado de enfermagem à criança. Os termos que emergem na primeira periferia são respectivamente meio e resposta ao desenvolvimento do cuidado.

O planejamento da assistência é uma imposição legal ${ }^{(16)}$. Ao planejar o cuidado, o enfermeiro incorpora a responsabilidade da assistência. O planejamento permite diagnosticar as necessidades do cliente, elaborar prescrições adequadas de cuidados, orientar a supervisão do desempenho do pessoal e a avaliar dos resultados ${ }^{(3,19)}$.

Portanto, o planejamento do cuidado gera qualidade assistencial. A qualidade pode ser observada e mensurada quando o planejamento do cuidado é fundamentado na cientificidade, nas experiências individuais e coletivas, e nas constantes construção e reconstrução dos processos ${ }^{(3,5)}$.

Desta forma, a SAE é importante ferramenta da gestão da assistência, servindo de base para o planejamento do cuidado desde a implementação dos manuais de normas e rotinas das unidades, padronização dos procedimentos, até a adoção do $P E^{(5,18)}$.

Os termos que emergem na segunda periferia (quadrante inferior direito), "assistência", "compromisso", "processo" e "responsabilidade", organizam a RS dos enfermeiros acerca da SAE, dando sustentação ao núcleo central da representação que é o cuidado. São apontadas neste quadrante palavras que valorizam aspectos ligados à dimensão atitudinal do indivíduo, como requisitos importantes para o exercício do cuidado.

As palavras "assistência" e "processo" estabelecem relação proximal como caminho a ser seguido, ou seja, as dinâmicas e as estratégias que o auxiliam e fortalecem o processo de aplicação da SAE no cuidado.

A prática de enfermagem diferenciada à criança requer o envolvimento de ações de cuidado direto e indireto. As ações diretas estão relacionadas ao operacional do cuidado - condições voltadas à prescrição e ao desenvolvimento do cuidado em si.

As ações indiretas estão relacionadas ao perfil do cuidador, e devem organizar e individualizar a sua assistência, com base no seu compromisso com os valores éticos e morais, a percepção que este tem do Estatuto da Criança e do Adolescente, o conhecimento das políticas de apoio à criança em todas as suas fases da vida e, sobretudo, o empenho e a responsabilidade que este esboça no processo de cuidar.

A zona de contraste (quadrante inferior esquerdo) corresponde a palavras que foram evocadas e estão relacionadas ao núcleo central, porém obtiveram ordem média de evocações e distribuição com valores baixos. Com relação ao termo indutor "Sistematização da Assistência de Enfermagem", as palavras "ciência", "humanização" e "obrigação" traduzem avaliação positiva da SAE.

A sistematização do cuidado por meio da rigorosidade metódica expressa a ciência da enfermagem. A aproximação da SAE à estratégia de humanização do cuidado de enfermagem modifica o ambiente hospitalar, vinculando os avanços científicos da área às individualidades do cliente, permeando o processo assistencial por atitudes humanísticas ${ }^{(19)}$. 
Entretanto, ainda neste quadrante, a palavra "obrigação" associa o desenvolvimento do cuidado sistematizado à tendência negativa, pois o desenvolvimento da SAE constitui parte da ciência do enfermeiro. A aproximação da SAE ao conceito de obrigação pode estar atrelada à frágil visão do processo de trabalho da enfermagem e da autonomia deste profissional, já que as demandas assistências deveriam ser fortalecidas por esta metodologia.

\section{CONSIDERAÇÕES FINAIS}

Os resultados sugerem que, para os enfermeiros que participaram do estudo, o núcleo central das representações sociais guarda estreita relação com o conceito de Sistematização da Assistência de Enfermagem, sendo representada pelo próprio cuidado.

Os núcleos central e periférico estão em consonância com a metodologia de organização e sistematização do cuidado, com base nos princípios do método científico da Sistematização da Assistência de Enfermagem, que buscam orientar o modelo de organização, visando administrar os diversos recursos necessários para o desenvolvimento do cuidado.

De acordo com as evocações dos enfermeiros, a centralidade "o cuidado de enfermagem" prestado à criança não pode prescindir de organização, planejamento, qualidade, comprometimento, responsabilidade e a consideração de ação processual.

Ressalta-se que a aplicação da Sistematização da Assistência de Enfermagem, no atendimento infantil, contribui de maneira positiva para o acolhimento efetivo da criança e sua família na trajetória da internação hospitalar. Percebe-se a importância da compreensão do cuidado como práxis do enfermeiro e do aprimoramento do conhecimento científico como estratégia de ampliação da autonomia profissional.

Embora ciente de que uma investigação desta natureza não se presta a obtenção de resultados conclusivos, espera-se ter contribuído para o aclaramento deste importante construto da ciência da Enfermagem.

\section{REFERÊNCIAS}

1. Waldow VR. Enfermería: la práctica del cuidado desde un punto de vista filosófico. Investig. Enferm. Imagen Desarr. [Internet] 2015;17(1) [acesso em 30 set 2016]. Disponível: http://dx.doi.org/10.11144/Javeriana.IE17-1.epdc.

2. Salvador PTCO, Santos VEP, Zeferino MT, Tourinho FSV, Vítor AF. Típico ideal de acadêmicos de enfermagem acerca da sistematização da assistência de enfermagem. Reme, Rev. Min. Enferm. [Internet] 2015;19(2) [acesso em 17 nov 2016]. Disponível: http://www.dx.doi.org/10.5935/1415-2762.20150025.

3. Casafus KCU, Dell'Acqua MCQ, Bocchi SCM. Entre o êxito e a frustração com a sistematização da Assistência de enfermagem. Esc. Anna Nery. [Internet] 2013;17(2) [acesso em 30 set 2016]. Disponível: http://dx.doi.org/10.1590/ S1414-81452013000200016.

4. Buboltz FL, da Silveira A, Neves ET. Estratégias de famílias de crianças atendidas em pronto-socorro pediátrico: a busca pela construção da integralidade. Texto Contexto Enferm. [Internet] 2015;24(4) [acesso em 30 set 2016]. Disponível: http://dx.doi.org/10.1590/0104-0707201500002040014.

5. da Silva TP, da Silva MM, Valadares GV, Silva IR, Leite JL. Nursing care management for children hospitalized with chronic conditions. Rev. bras. enferm. [Internet] 2015;68(4) [acesso em 17 nov 2016]. Disponível: http:// dx.doi.org/10.1590/0034-7167.2015680410i.

6. Moscovici S. O fenômeno das representações sociais. $5^{a}$ ed. Petrópolis: Vozes; 2003. Representações sociais: investigações em psicologia social p. 29-109.

7. Abric JC. A abordagem estrutural das representações sociais. In: Moreira AS, Oliveira DC, ed. Estudos interdisciplinares em representações sociais. Goiânia: AB Editora; 1998. p. 27-38. 
8. Borges MS, Queiroz LS, da Silva HCP. Representações sociais sobre cuidar e tratar: o olhar de pacientes e profissionais. Rev. esc. enferm. USP. [Internet] 2011;45(6) [acesso em 30 set 2016]. Disponível: http://dx.doi. org/10.1590/S0080-62342011000600021.

9. Reis AOA, Sarubbi Junior V, Bertolino Neto MM, Rolim Neto ML. Tecnologias computacionais para o auxilio em pesquisa qualitativa: Software EVOC. São Paulo: Schoba; 2013.

10. Collière MF. Promover a vida: da prática das mulheres de virtude aos cuidados de enfermagem. $5^{\mathrm{a}}$ ed. Lisboa: Lidel; 1999.

11. Abud SM, Gaíva MAM. Registro dos dados de crescimento e desenvolvimento na caderneta de saúde da criança. Rev. Gaúcha Enferm. [Internet] 2015;36(2) [acesso em 30 set 2016]. Disponível: http://dx.doi.org/10.1590/19831447.2015.02.48427.

12. Rodrigues PF, Amador DD, Silva KL, Reichert APS, Collet N. Interação entre equipe de enfermagem e família na percepção dos familiares de crianças com doenças crônicas. Esc. Anna Nery. [Internet] 2013;17(4) [acesso em 30 set 2016]. Disponível: http://dx.doi.org/ 10.5935/1414-8145.20130024.

13. Marques CDC, Lima MF, Malaquias TSM, Waidman MAP, Higarashi IH. O cuidador familiar da criança hospitalizada na visão da equipe de enfermagem. Cienc Cuid Saude. 2014;13(3):541-8.

14. Borges MS, dos Santos DS. O campo de cuidar: uma abordagem quântica e transpessoal do cuidado de enfermagem. Cienc Cuid Saude. [Internet] 2013;12(3) [acesso em 30 set 2016]. Disponível: http://dx.doi. org/10.4025/cienccuidsaude.v12i3.17159.

15. Alves EATD, Cogo ALP. Percepção de estudantes de enfermagem sobre o processo de aprendizagem em ambiente hospitalar. Rev. Gaúcha Enferm. [Internet] 2014;35(1) [acesso em 30 set 2016]. Disponível: http://dx.doi. org/10.1590/1983-1447.2014.01.42870.

16. Brasil. Lei n. 7.498, de 25 de junho de 1986. Dispõe sobre a regulamentação do Exercício da Enfermagem e dá outras providências. Diário Oficial da República Federativa do Brasil, Brasília, 26 jun. 1986. Seção 1:1.

17. Meira MDD, Kurcgant P. Nursing education: training evaluation by graduates, employers and teachers. Rev. bras. enferm. [Internet] 2016;69(1) [acesso em 30 set 2016]. Disponível: http://dx.doi.org/10.1590/00347167.2016690102i.

18. Vituri DW, Évora YDM. Total Quality Management and hospital nursing: an integrative literature review. Rev. bras. enferm. [Internet] 2015;68(5) [acesso em 30 set 2016]. Disponível: http://dx.doi.org/10.1590/00347167.2015680525i.

19. Rodrigues AC, Calegari T. Humanização da assistência na unidade de terapia intensiva pediátrica: perspectiva da equipe de enfermagem. Reme, Rev. Min. Enferm. [Internet] 2016;(20) [acesso em 30 set 2016]. Disponível: http://www.dx.doi.org/10.5935/1415-2762.20160003. 